\title{
CLAVES PARA UNA ENTREVISTA \\ CON VÍCTIMAS DE ABUSO \\ SEXUAL EN LA INFANCIA
}

\author{
CARLOTA BASTIDA MERA ${ }^{1}$ \\ MARÍA PRIETO URSÚA ${ }^{2}$
}

Fecha de recepción: junio de 2020

Fecha de aceptación y versión definitiva: septiembre de 2020

\begin{abstract}
RESUMEN: Con este trabajo se pretende realizar una revisión exhaustiva de los posibles efectos derivados de sufrir una experiencia de abuso sexual y las variables influyentes. El principal objetivo de este estudio es identificar las claves que debe tener una entrevista de evaluación para estas víctimas, revisando los principales protocolos existentes y tratando de extraer las características esenciales para lograr una técnica que permita obtener la máxima información posible causando el menor daño a la víctima, ya que la máxima de toda intervención con víctimas debe ser siempre evitar la revictimización.
\end{abstract}

PALABRAS CLAVE: violencia sexual; abuso sexual infantil; efectos; entrevista; revictimización.

\section{Keys to Interviewing Victims of Child Sexual Abuse}

ABSTRACT: The aim of this work is to carry out an exhaustive review of the effects that an experience of sexual abuse can have on victims and the variables that influence them. The main objective of this study is to identify the keys that an assessment interview with these victims should have, reviewing the main existing protocols and trying to extract the essential characteristics to achieve a technique that allows the maximum information possible to be obtained causing the least harm to the victim, since the maxim of any intervention with victims should always be to avoid re-victimization.

KEY WORDS: sexual violence; child sexual abuse; effects; interview; re-victimization.

1 Estudiante titulada del doble grado en Psicología y Criminología. Universidad Pontificia Comillas. Correo electrónico: carlotabmera@gmail.com.

2 Profesora del departamento de Psicología. Universidad Pontificia Comillas. Correo electrónico: mprieto@comillas.edu. 


\section{INTRODUCCIÓN}

El abuso sexual infantil (ASI) se define como «cualquier conducta sexual mantenida entre dos personas, al menos una de ellas menor, entre las que existe una situación de desigualdad, ya sea por razones de edad o de poder, y en las que el menor es utilizado para la estimulación sexual de otra persona» (Centros de Servicios Sociales y Servicio Especializado en Menores, 2014). El abuso sexual a menores podría entenderse como cualquier tipo de conducta sexuai mianitenida entre un adulto y un menor (Echeburúa y Corral, 2006).

La victimización sexual de los menores es un problema de especial gravedad tanto en el nivel mundial como en el nacional (Pereda, 2016). El estudio de los abusos sexuales hacia los menores ha adquirido, en las últimas décadas, mucho protagonismo en Estados Unidos, Canadá y todo el mundo anglosajón, en general, así como en los países del norte de Europa, fundamentalmente (López, 1996).

Conseguir una medida verdaderamente fiable de la frecuencia con la que ocurren estos delitos es muy difícil, ya que muchos casos permanecen ocultos durante un largo período de tiempo o nunca llegan a ser denunciados (De la Cruz, 2014). En el estudio de la relevancia que tienen este tipo de delitos en el panorama actual vamos a trabajar con datos provenientes de distintos tipos de estudios: estudios de incidencia y estudios de prevalencia. Teniendo en cuenta que un alto porcentaje de los casos ocurridos no son denunciados en el momento que ocurre la agresión, sino que son revelados con posterioridad, será esperable encontrar porcentajes significativamente inferiores en los estudios de incidencia que en los de prevalencia (Pereda, 2016).

Numerosos estudios nacionales e internacionales constatan la fuerte presencia de este problema en el mundo occidental, con una prevalencia de entre un 10\% y un 20\% de la población (Pereda y Gallardo-Pujol, 2011). Según Pereda (2016), distintos estudios de meta-análisis sitúan los porcentajes de victimización sexual a menores alrededor del $8 \%$ en los varones y cerca del $20 \%$ en las mujeres, en el nivel mundial.

En España, López (1995) realiza el primer estudio acerca de la incidencia de los delitos de abuso sexual a menores, cuyos resultados muestran la elevada tasa de incidencia de abusos sexuales a menores: un 15\% de hombres y $22 \%$ de mujeres habían sido víctimas de abuso sexual en nuestro país antes de cumplir los 18 años. Cantón-Cortés y Justicia (2008) realizan un estudio con una muestra de menores españoles de 13 años cuyos resultados muestran una prevalencia de victimización del 6,5\% en hombres y $10 \%$ en mujeres. 
Según estas cifras tan significativas, podemos considerar el abuso sexual infantil como un fenómeno alarmantemente presente en la realidad actual que merece una atención especial.

A la hora de definir el concepto de abuso sexual, los profesionales del campo de la salud ofrecen una definición mucho más amplia y menos restrictiva que las definiciones que ofrecen los profesionales del campo del derecho (De la Cruz, 2014). El Código Penal de nuestro país dispone lo siguiente en su artículo 181.1: «El que, sin violencia o intimidación y sin que medie consentimiento, realizare actos que atenten contra la libertad o indemnidad sexual de otra persona, será castigado, como responsable de abuso sexual [...]». Desde el punto de vista legal, se entiende que no hay consentimiento cuando la víctima padece un trastorno mental grave, tiene su voluntad anulada por el consumo de cualquier tipo de sustancia o fármaco, está privada del sentido o cualquier otra situación que coloque al agresor en una posición de evidente superioridad frente a la víctima.

Cuando hablamos de abuso sexual dirigido a menores, desde el punto de vista psicológico, nos vamos a encontrar discrepancias entre los autores a la hora de describir este fenómeno (Turcios, 2012). Encontramos diferencias, fundamentalmente, a la hora de determinar rangos de edad dentro de los cuales una agresión de este tipo debería considerarse «abuso sexual infantil» (López, 1995) y en la descripción de las conductas o niveles de conducta que se enmarcan en el concepto de «abuso» (Mas, 1995). Sin embargo, según la literatura, vamos a considerar dos criterios fundamentales y suficientes para definir el abuso sexual infantil (ASI) en este ámbito: coerción y asimetría (Carrasco y Maza, 2005; Echeburúa y Corral, 2006; De la Cruz, 2014; López, 1996). La coerción se entiende como la capacidad que tiene el abusador de ejercer presión sobre el menor, explícita o implícita, es decir, tanto física como psicológica o mediante engaño, y la asimetría hace referencia a la diferencia significativa de edad entre abusador y víctima, que impide cualquier posibilidad de libre consentimiento por parte del menor (De la Cruz, 2014).

Con todo ello, podría establecerse el límite a partir del cual vamos a hablar de abuso sexual en el momento en que el menor pierde el control sobre su propia sexualidad, convirtiéndose en un instrumento de placer o estimulación sexual para otro (Turcios, 2012).

Lo más importante a la hora de acercarnos al fenómeno del abuso sexual, más allá de su alta prevalencia, es que supone una experiencia traumática para quien lo sufre, provocando graves consecuencias negativas sobre el funcionamiento psicológico de la víctima, así como la predisposición a sufrir alteraciones emocionales o desarrollar comportamientos sexuales inadaptados en la vida adulta (Echeburúa y Corral, 2006). 
Son numerosos los efectos que tiene sobre la víctima una experiencia de abuso sexual, tanto en el corto plazo como en el largo plazo, y, a su vez, podrán ser muy diversos, ya que la experiencia de cada uno es única y particular (Burgess y Holmstrom, 1974).

En rasgos generales, sentimientos de desconfianza, miedo, asco y hostilidad hacia el agresor, vergüenza y ansiedad serían claros indicadores en el corto plazo de que se ha cometido un abuso sexual, además de los indicadores físicos (López, 1995).

Los efectos a largo plazo son menos frecuentes, pueden afectar alrededor de un 30\% de las víctimas (Echeburúa y Corral, 2006). En líneas generales, el haber sido víctima de ASI va a influir en el desarrollo de la persona en diferentes esferas, favoreciendo el padecimiento de trastornos emocionales como la depresión, la ansiedad, baja autoestima y dificultades en las relaciones interpersonales en la adultez, así como trastornos disociativos y de personalidad e incluso Trastorno de Estrés Post-Traumático (Cantón-Cortés y Cortés, 2015) y con mucha frecuencia aparece relacionado con un historial de abuso de alcohol, drogas y conductas delictivas (Ullman, Najdowski y Filipas, 2009).

Sin embargo, no podemos olvidar que no todas las víctimas van a reaccionar igual y esto se debe a la influencia de distintas variables cuya presencia o ausencia va a influir sobre la intensidad de los efectos que esta experiencia pueda tener sobre la víctima (Burgess y Holmstrom, 1974). A la hora de modular la gravedad de las consecuencias de esta victimización, vamos a distinguir entre variables que intensifican los efectos en la víctima y variables que favorecen un menor impacto sobre la misma (Echeburúa y Corral, 2006).

La elevada tasa de prevalencia y los efectos que tiene sobre las víctimas hacen del abuso sexual una problemática que merece especial atención y sobre todo hace cuanto menos aconsejable el desarrollo de programas de prevención y atención para las víctimas (López, 1995).

Existen una gran cantidad de protocolos diversos a la hora de intervenir con víctimas. Según la literatura, los más utilizados con víctimas de abuso sexual son, en caso de víctimas menores en el momento de la evaluación: el Protocolo NICHD, el Protocolo de entrevista forense de Michigan, el Protocolo RATAC-SATAC, la entrevista step wise o paso a paso, la entrevista asistida para la evaluación del abuso sexual infantil EASI-5, la entrevista de evaluación del testimonio infantil EATI; y con adultos: la entrevista cognitiva, la entrevista semiestructurada sobre agresiones sexuales o la entrevista clínica-forense (Vizcarra, Marin y Díaz, 2018).

Distintos estudios internacionales han destacado la importancia de los protocolos de entrevista en la evaluación a víctimas, ya que permiten recabar 
la máxima información posible y, al poder ser grabadas, evitan obligar a la víctima a pasar por el mismo proceso varias veces, reduciendo la revictimización (Lama et al., 2015). Es por ello por lo que este trabajo se va a centrar en identificar las claves de la entrevista con víctimas de ASI que garanticen una exploración exhaustiva de los hechos y, al mismo tiempo, una satisfacción de las necesidades de la víctima entrevistada, evitando la revictimización.

\section{OBJETIVOS Y METODOLOGÍA}

El principal objetivo que se persigue con la realización de este trabajo es identificar las características o claves fundamentales de una entrevista con víctimas de agresión sexual, que permitan un conocimiento profundo de los hechos sin causar más daño en la interacción con las víctimas.

Los objetivos específicos son elaborar una descripción de los efectos de la agresión sexual, tanto en el corto como en el largo plazo, identificar los riesgos para las víctimas asociados a la realización de este tipo de entrevistas y diferenciar las características básicas de una entrevista de evaluación de las características específicas que debe presentar una entrevista con víctimas de abuso sexual.

Para ello se ha realizado una revisión bibliográfica con la finalidad de conocer el estado de la cuestión planteada en el panorama científico actual. Se trata de un estudio fundamentalmente inductivo, orientado a la revisión descriptiva de la teoría y los modelos teóricos vigentes con el objetivo de lograr una visión panorámica de la problemática objeto de estudio.

La revisión incluyó investigaciones empíricas publicadas, tanto cualitativas como cuantitativas, centradas en los efectos del abuso sexual infantil para las víctimas, las variables mediadoras, las técnicas de evaluación y el fenómeno de revictimización, fundamentalmente. Los estudios seleccionados estaban publicados mayoritariamente en inglés, aunque también se incluyeron en la búsqueda estudios nacionales; estaban publicados en revistas científicas; no se aplicaron filtros de fechas, dando aun así prioridad en la selección de los artículos a aquellos más recientes frente a los más anteriores; también se seleccionaron prioritariamente aquellos artículos disponibles en texto completo, aunque algunos que fueron considerados fundamentales se solicitaron a los propios autores a través de ResearchGate; finalmente, se utilizaron también libros completos extraídos de la colección de la biblioteca de la Universidad Pontificia Comillas de Cantoblanco y Alberto Aguilera. 
Se recurrieron a distintas bases de datos interdisciplinares para garantizar una cobertura más amplia. Las bases de datos de las cuales se obtuvieron los distintos documentos utilizados para la realización de este estudio fueron: Psychinfo, Psicodoc, Scopus, Web of Science, Academic Search Complete, Dialnet y la herramienta de búsqueda online Discovery para la Universidad Pontificia Comillas, así como su base de datos y el repositorio de su biblioteca.

Dentro de estas bases de datos se realizaron diversas búsquedas avanzadas aplicando distintos criterios:

(Entrevista OR evaluación) AND (víctimas OR «víctimas de abuso sexual» OR «abuso sexual infantil»)

«Child sexual abuse» * victims * effects * «long term» * «short term» * «interview keys»

Quedaron fuera de la selección los artículos que hacían referencia a la entrevista con víctimas menores en el momento de la agresión o en momentos inmediatamente posteriores o la entrevista forense para comprobar si se ha cometido verdaderamente el abuso sexual o no.

\section{RESULTADOS}

\subsection{Efectos del abuso SEXUAL EN LAS víctimas}

El abuso sexual infantil ha demostrado tener graves consecuencias negativas para quién lo sufre, causando diversa sintomatología que varía de unas víctimas a otras tanto en su presencia o ausencia como en su intensidad. Además, el ASI, debe considerarse como un factor de riesgo para el desarrollo de psicopatologías tanto en la infancia como en la adultez, ya que va a tener un fuerte impacto sobre el desarrollo de los sujetos en diferentes áreas (Lama et al., 2015). Las diferentes reacciones que podemos observar en quienes han sido víctimas de abuso en la infancia, además, predisponen o aumentan el riesgo de volver a ser víctimas de abuso en la adultez (Ullman y Vasquez, 2015).

\section{En el momento de la agresión}

En los momentos inmediatamente posteriores a la agresión o experiencia traumática, el ASI va a provocar efectos que disminuyen, evolucionan o se transforman en el largo plazo. 
El abuso sexual puede tener consecuencias físicas sobre la víctima como lesiones específicas en las localizaciones genital, anal, oral o los senos, así como lesiones físicas añadidas producidas o bien como respuesta a la resistencia de la víctima o bien de forma intencionada con el fin de causar más daño o terminar con la vida de la víctima. Este tipo de agresiones también pueden suponer el contagio de una enfermedad de transmisión sexual o incluso un embarazo, cuando la víctima es una mujer (De la Cruz, 2014; López, 1996).

Toda experiencia de abuso sexual va a provocar reacciones emocionales negativas en las víctimas. Las más inmediatas que suelen aparecer con más frecuencia son la sensación de irrealidad, el miedo intenso, sentimientos de vergüenza y culpa, baja autoestima y pérdida de confianza en uno mismo. En el nivel cognitivo, es frecuente la disminución de la concentración, el procesamiento de información y la toma decisiones. Todos estos efectos se traducen en conductas como rabia o llanto y comportamientos de indefensión aprendida (De la Cruz 2014; González y Pardo, 2007; López, 1996).

Las víctimas de ASI también van a sufrir consecuencias en su funcionamiento social y alteraciones en su vida cotidiana, provocando déficits en la interacción y relaciones interpersonales y aumentando la probabilidad de que la víctima realice conductas de riesgo como el consumo de sustancias, comportamiento antisocial o conductas sexuales anormales (De la Cruz, 1014; López, 1996).

Desde el punto de vista neurobiológico, la infancia es un período crítico de desarrollo en el cual la experiencia de situaciones traumáticas, como el ASI, va a suponer alteraciones en distintos niveles que aumentan la probabilidad de desarrollar trastornos psicológicos de adulto. Las afecciones relacionadas con sufrir abuso sexual en la infancia son: alteraciones en el eje neuroendocrino hipotalámico-hipofisario adrenal, relacionado con el desarrollo de trastornos de ansiedad, depresión y estrés post-traumático; alteraciones en el desarrollo de distintas estructuras cerebrales, lo que está relacionado con el desarrollo de la personalidad; o un déficit en la comunicación o conectividad entre las distintas estructuras cerebrales, que perjudica el aprendizaje y afecta al funcionamiento cerebral los estímulos de miedo, por ejemplo. A todas estas afecciones debemos añadir las alteraciones neuropsicológicas que suponen un déficit en los procesos básicos de atención, concentración y memoria (Pereda y Gallardo-Pujol, 2011).

A grandes rasgos, una experiencia de abuso sexual normalmente se va a traducir en una peor salud física y, sobre todo, una peor percepción de la propia salud (López, 1996). 
En el largo plazo

Tras la fase crítica, existe un período de reorganización donde la víctima puede recuperar su funcionamiento previo. Sin embargo, cuando esta reorganización no se alcanza, podemos ver en las víctimas efectos muy variados en el largo plazo que dependen de la influencia de numerosas variables mediadoras y factores intermedios que determinarán el curso de la sintomatología en cada uno de los sujetos (López, 1996).

Los efectos que suelen aparecer en las víctimas diferidos en el tiempo están más cronificados. Los más frecuentes son los trastornos adaptativos de ansiedad o depresión, el Trastorno de Estrés Post-Traumático, trastornos disociativos, alteraciones permanentes en la personalidad, disfunciones sexuales y la adquisición de hábitos de mala salud como el consumo de drogas y alcohol o la realización de conductas sexuales de riesgo (González y Pardo, 2007). La predisposición a realizar conductas de riesgo convierte a las víctimas en sujetos más vulnerables o susceptibles de volver a convertirse en víctimas en el futuro (De la Cruz, 2014).

Es muy frecuente después de sufrir un abuso sexual que las víctimas realicen antes o después importantes cambios en su vida, como el cambio de residencia o de trabajo (Dos Reis, Baena de Moraes y Duarte, 2016) ya que la literatura relaciona fuertemente la experiencia de abuso con un peor ajuste social y laboral, dificultades relaciones y una alteración de la vida cotidiana de las víctimas (De la Cruz, 2014; López, 1996).

Duma, Mekwa y Denny (2007) describen, a partir de un estudio llevado a cabo con víctimas de abuso sexual en Sudáfrica, el siguiente proceso de recuperación en 8 pasos: (1). Trauma sexual, (2). Despertar, (3). Aceptación pragmática, (4). Punto de inflexión, (5). Reclamar lo que se ha perdido, (6). Definir los propios objetivos de recuperación, (7). Estar preparado para el cierre y (8). Vuelta a uno mismo. Este proceso de superación también se va a ver afectado tanto positiva como negativamente en función de la respuesta recibida por parte de las personas más relevantes del entorno de la víctima: familiares, amigos y servicios especializados (Dos Reis et al., 2016).

\subsection{VARIABLES INFLUYENTES}

No debemos olvidar que no existe una sintomatología común o un cuadro aplicable a todas las víctimas de abuso sexual, sino que cada una presenta una vivencia única y particular con unos efectos característicos marcados por distintas variables moduladoras que pueden o bien, reducir los efectos negativos sobre las víctimas, o bien, intensificarlos. 
De las variables que van a amortiguar los efectos del ASI sobre las víctimas habría que diferenciar, según González y Pardo (2007), por un lado, variables individuales, intrínsecas al propio sujeto que sufre la experiencia traumática, y, por otro lado, variables extrínsecas que subyacen a dicha experiencia.

En cuanto a las variables individuales, diversos estudios confirman que tener altas capacidades verbales, relacionadas con la capacidad de asociación, permiten a los sujetos resignificar su experiencia, ponerla en palabras y expresarla o transmitirla a los demás, esto es, sacarla fuera de sí mismos, lo que reduce el impacto. Un buen desarrollo de las funciones ejecutivas, que implica la capacidad de planificar y ordenar la conducta hacia unos objetivos marcados anticipando posibles consecuencias, también funciona como factor de protección en las víctimas (González y Pardo, 2007). Por último, habría que añadir en este nivel, disponer de distintas estrategias de afrontamiento, capacidad de procesamiento y flexibilidad mental (Lama et. al, 2015). Además, como algunos efectos neuropsicológicos vienen determinados genéticamente, ser portador de determinados alelos también podría considerarse como una protección para los sujetos ante los efectos que el ASI produce en el desarrollo del cerebro, sobre todo en etapas tempranas del desarrollo (Pereda y Gallardo-Pujol, 2011).

Por el contrario, vamos a ver cómo los sentimientos de vergüenza y culpa hacia el suceso traumático y los pensamientos mal-adaptativos o creencias disfuncionales en relación con el abuso sexual intensifican las consecuencias negativas para las víctimas. Los sentimientos de vergüenza y culpa son mediadores fundamentales entre la agresión y la sintomatología, siendo especialmente relevantes a la hora de valorar la intensidad de los síntomas en las víctimas (Shin, Chang, Cho, Kim, Kim y Chung, 2015).

No debemos olvidar hacer alusión a la edad como variable relevante, ya que va a influir en el significado que la víctima le atribuye al suceso, siendo más traumático a mayor edad, normalmente (González y Pardo, 2007). Esto se debe a que en víctimas de mayor edad es esperable una mayor capacidad cognitiva que favorece la comprensión de lo que verdaderamente está sucediendo (Echeburúa y Corral, 2006). Además, la edad va a marcar la tendencia a presentar una sintomatología u otra, siendo el síntoma más característico en víctimas de edad preescolar la aparición de comportamientos sexuales inapropiados, mientras que en víctimas de edad escolar y adolescente destaca la presencia de sintomatología externalizante, trastornos disociativos, problemas en las relaciones con iguales, entre otros (Cantón-Cortés y Cortés, 2015). 
Por otro lado, atendiendo a los factores extrínsecos, han demostrado ser los más influyentes a la hora de proteger a la víctima del impacto del ASI los siguientes: la existencia de una figura de referencia y apoyo en el momento de revelar el suceso, la reacción del entorno y, sobre todo, la intervención y asistencia terapéutica temprana. Las propias víctimas reconocen este último como el factor más importante a la hora de superar la experiencia traumática (Burgess y Holmstrom, 1974; Dos Reis et al., 2016; González y Pardo, 2007; Lama et al., 2015). La falta de apoyo o desconfianza es la variable más determinante en relación con la intensidad de los efectos del ASI, suponiendo el apoyo de las personas cercanas el factor de protección más importante en casos de este tipo (Stiller y Hellman, 2017).

Las variables que, a lo largo de la literatura, más se han relacionado con un impacto mayor o consecuencias más negativas del abuso sexual infantil sobre las víctimas son, fundamentalmente, las características del acto en sí que lo convierten en una experiencia más intensa o dañina (como, por ejemplo, el que haya penetración frente a que no la haya, la presencia de otras violencias añadidas al acto sexual, la causación de lesiones físicas o la percepción de riesgo para la propia vida). El padecimiento de trastornos psicopatológicos previos como la ansiedad o depresión o una historia previa de traumas en la víctima también va a hacer la experiencia traumática más perjudicial para la víctima (González y Pardo, 2007). También lo serían la existencia de una relación previa entre víctima y agresor y las consecuencias negativas derivadas de la revelación del abuso. En relación con estas dos variables, cuando víctima y agresor tienen una relación previa de cualquier tipo (familiar, amigo de la familia, amigo de la víctima...) la revelación del suceso coloca a la víctima en una posición de conflicto que puede generar otros traumas asociados, como, por ejemplo, que se rompa la familia. En esta línea, cabe mencionar también el grado de culpabilización de la víctima por lo sucedido, que supone un riesgo mayor cuando es alta (Echeburúa y Corral, 2006). Finalmente, la falta de apoyo o asistencia inmediata se ha confirmado como el factor más influyente sobre los efectos de la experiencia traumática en las víctimas (Burgess y Holmstrom, 1974).

\subsection{RIESGOS ASOCIADOS A LA ENTREVISTA: LA REVICTIMIZACIÓN}

Este fenómeno hace referencia al conjunto de costes personales que sufre la persona como consecuencia de una intervención estatal inadecuada (Muñoz, 2013). 
La revictimización o victimización secundaria debe definirse como el acto de recrear o reincorporar forzosamente los elementos del delito de agresión sexual u ofensa sexual de gravedad penal a través de las etapas judiciales que afecten neurológica, psicológica o emocionalmente a la víctima del dolo in importar el sexo, edad, raza o condiciones sociodemográficas victimales. Este proceso se caracteriza por la incapacidad de la víctima de defenderse ante la Ley y la impotencia de adquirir Justicia, resultando la víctima en el ser perjudicado y su victimario protegido y amparado por la Ley (Mercado, 2017).

El simple hecho de hablar sobre lo que ha ocurrido va a generar sentimientos en la víctima que pueden favorecer o perjudicar la integración de la experiencia en función de la reacción del entorno (Stiller y Hellmann, 2017), por eso es fundamental para evitar la revictimización que la víctima no se sienta juzgada y sienta que es creída y protegida. Priorizar la obtención de información en la entrevista frente a la atención personal a la víctima va a aumentar dichos sentimientos negativos (Muñoz, 2013).

La respuesta que recibe la víctima de su entorno más cercano va a influir fundamentalmente en los sentimientos de culpa y vergüenza, que están íntimamente relacionados con la intensidad de los efectos negativos en las víctimas de abuso sexual (Dos Reis et al., 2016). Estos efectos se verán intensificados ante una experiencia de revictimización (Cantón-Cortés y Cortés, 2015).

La experiencia negativa por parte de la víctima del proceso judicial va a obstaculizar también el proceso de superación (Lama et al., 2015). Para evitar esto, es necesario garantizar que las víctimas dispongan de toda la información acerca del proceso y de los profesionales que van a intervenir, así como asegurar un entorno de intimidad y protección para las entrevistas donde la víctima no se sienta cuestionada ni puesta en duda (Muñoz, 2013).

Las reacciones de la víctima tras la experiencia de abuso también pueden favorecer la revictimización. Se ha observado que cuando las víctimas ponen en marcha estrategias desadaptativas de afrontamiento, aumentan la probabilidad de revictimización (Cantón-Cortés y Justicia, 2008). Conductas de evitación como el aislamiento, el consumo de drogas o alcohol, la realización de conductas de riesgo y el aumento de las conductas sexuales favorecen la revictimización ya que retrasan el afrontamiento y la confrontación con el evento traumático que se ha vivido, provocando que los efectos que se habían tratado de evitar se experimenten diferidos en el tiempo y, normalmente, con mayor intensidad (Filipas y Ullman, 2006). En esta línea, la desregulación emocional, que se entiende como la dificultad para manejar y responder a las propias emociones, se ha relacionado directamente con un mayor riesgo 
de sufrir revictimización, debido a que impide la puesta en marcha de comportamientos autoprotectores (Ullman y Vasquez, 2015).

Los efectos de la revictimización pueden ser más traumáticos y perniciosos que las circunstancias de la victimización primaria y van a agravar las aflicciones neuropsicoemocionales en la víctima (Mercado, 2017), e incluso la sintomatología puede aparecer en la edad adulta a causa de una revictimización (Cantón-Cortés y Cortés, 2015).

\subsection{Características bÁsicas de UnA ENTREVISTA DE EVALUACióN}

«La evaluación psicológica es aquella disciplina de la psicología científica que se ocupa de la exploración y análisis del comportamiento, en distintos niveles de complejidad, de un sujeto humano, o grupo especificado de sujetos, con distintos objetivos aplicados» (Fernández-Ballesteros, 2004, p. 23).

Toda evaluación psicológica debe ser un proceso estructurado y replicable, basado en la formulación y contraste de hipótesis y que conlleve una toma de decisiones para llegar a la solución del problema que se está evaluando (Muñoz, 2013).

Siempre que se realiza una entrevista de evaluación hay que tener en cuenta que la persona que tenemos delante tiene unas características individuales que la diferencian de otros y que marcan el patrón de su comportamiento (Fear, 1979), por ello es necesario conocer su historia, para poder ajustarnos al marco de referencia más adecuado para guiar la evaluación en cada caso.

En toda entrevista de evaluación hay que lograr que la persona se sienta cómoda para hablar espontáneamente de su historia. Para ello es necesario llevarla a cabo en un ambiente físico adecuado, que garantice la intimidad; y lograr un vínculo positivo con la persona entrevistada (FernándezBallesteros, 2004). Por otro lado, es necesario reconocer la importancia de las preguntas exploratorias, que nos permiten obtener la máxima información sobre los aspectos concretos que más interesan. Sin embargo, estas preguntas deben formularse siempre de modo que no resulten invasivas ni demasiado directas, se deben plantear de tal modo que no se altere el clima de una conversación agradable, manteniendo la espontaneidad en el sujeto entrevistado (Fear, 1979).

Lo más importante siempre en una entrevista de evaluación es la colaboración de quien está siendo entrevistado. Para lograr esta actitud en la persona, es necesario que el entrevistador muestre una actitud de preocupación y consideración de sus intereses, así como calidez o simpatía, sensibilidad y 
madurez. Además de estas actitudes, todo buen entrevistador debe ser capaz de adaptarse a las distintas situaciones, tener un pensamiento analítico y juicio crítico y ser capaz de mantener el control y guiar la entrevista en todo momento (Fear, 1979).

\subsection{CARACTERísticas de la ATENCión a víctimas de ABUSO SEXUAL INFANTIL}

Siempre que trabajemos con víctimas que han sufrido un abuso sexual en la infancia, se deben tener en cuenta las características diferenciales que van a marcar la situación, diferenciándola de cualquier otro tipo de entrevista de evaluación.

Las víctimas de este delito concreto, ASI, se consideran especialmente vulnerables a la hora de revivir su experiencia, ya que el trauma sexual es considerado como más sensible en comparación con otros debido a la intensidad de las emociones asociadas, así como la posible presencia de sentimientos de culpa y vergüenza asociados, que aparecen con más frecuencia en casos de trauma sexual que en cualquier otro (Nielsen, Hansen, Elklit y Bramsen, 2016).

La persona, en este caso, ha sufrido una situación traumática, si no dos (ya que si ha pasado por un procedimiento judicial inadecuado podemos considerar esta experiencia como una segunda victimización); lo que supone un factor que puede distorsionar los datos aportados en la entrevista (Muñoz, 2013). La revictimización es un factor que puede afectar a la exactitud de un relato sobre una situación vivenciada (Muñoz et al., 2016).

Son varios los estudios internacionales que apoyan el uso de protocolos de entrevista a la hora de evaluar a víctimas ya que ofrecen grandes ventajas tanto para el proceso, garantizando la obtención de la máxima información, como para la víctima, reduciendo el efecto de la revictimización ya que, al poder ser grabadas en vídeo, liberan a la víctima de la obligación de pasar por el mismo proceso varias veces (Lama et al., 2015).

En los casos de abuso sexual infantil, cuando la víctima ya es adulta, el método más utilizado es la entrevista cognitiva de Fisher y Geiselman (1992). Esta técnica consiste, fundamentalmente, en el uso de cuatro técnicas generales de incremento de memoria: reconstruir los contextos físicos y personales que existieron en el momento del hecho, recuperando elementos secuenciales, perceptuales y emocionales; recuerdo libre; cambio de perspectiva; y, recuerdo inverso o desde distintos puntos de partida.

Estas técnicas permiten la obtención de un gran número de detalles en torno al suceso, pero, además, posteriormente los autores añaden a la 
entrevista técnicas para obtener información sobre los factores sociales y comunicativos: preguntas compatibles con el testigo; crear un buen clima emocional; transferir el control de la entrevista al testigo; y, crear imágenes mentales de detalles específicos. Estas técnicas sirvieron para mejorar el bienestar mental de los entrevistados, aumentando su sentimiento de control y confianza (Vizcarra et al., 2018).

Otras propuestas que merece la pena tener en cuenta a la hora de entrevistar adultos víctimas de ASI son la Entrevista Semiestructurada sobre Agresiones Sexuales de Echeburúa, Corral, Sarasua, Zubizarreta y Sauca (1994) y, también, la Entrevista Clínica-Forense de Arce y Fariña (2012).

La primera es un formato de entrevista que busca establecer de forma ordenada las características del abuso sexual mientras que la segunda consiste en una entrevista abierta de discurso libre que se centra en comparar los síntomas, pensamientos y conductas que tiene la víctima en el momento actual con el estado previo a que sucedieran los hechos. Mientras que la entrevista estructurada de Echeburúa et al. (1994) se centra en recabar primero información sobre las circunstancias en que sucedieron los hechos y después sobre las acciones y reacciones de la víctima, la entrevista clínica-forense de Arce y Fariña (2012) pone énfasis en la información relativa a las relaciones familiares, sociales y laborales de las víctimas y el impacto que ha podido tener la experiencia traumática sobre ellas (Vizcarra et al., 2018).

Normalmente, estas entrevistas se realizan en el marco de una investigación judicial, aunque este supuesto no es el objeto de nuestro trabajo. En estos casos, la entrevista cognitiva permitiría el análisis y valoración de la huella de memoria que suele convertirse en el principal medio de prueba ante la ausencia de otros indicios físicos u objetivos (Muñoz et al., 2016). A través de esta técnica se obtiene la prueba testifical, que constituye lo que se denomina «indicio cognitivo». Para garantizar la validez de la prueba es necesario que cumpla con los mismos estándares de conservación que se aplican a las pruebas físicas. La ley de Enjuiciamiento Criminal, en sus artículos 435-439 y 709, establece cómo se debe preguntar al testigo y qué preguntar no se deben hacer. Sin embargo, es importante tener en cuenta que es fundamental que los psicólogos encargados de abordar estos casos tengan una formación especializada para evitar sesgos que puedan contaminar la entrevista (Muñoz et al., 2016).

En el caso de víctimas menores de edad en el momento de la evaluación existen otros protocolos que buscan favorecer la espontaneidad del menor durante la entrevista y facilitar la obtención de la información verídica, como la entrevista Step Wise, el protocolo Michigan, el protocolo NITCH o el protocolo RATAC-SATAC, entre otros (Vizcarra et al., 2018). 
Aunque este supuesto tampoco se corresponde con el objetivo de este trabajo, que se centra en la evaluación psicológica de las víctimas en su adultez, merece la pena revisarlos y mencionar algunas ideas que puedan ser relevantes.

Estas propuestas presentan una estructura de entrevista muy parecida. Se recomienda siempre comenzar presentándonos ante el menor y explicándole como va a ser la entrevista. El principal objetivo es establecer una buena relación con el entrevistado, para lo que se recomienda comenzar hablando de gustos o intereses de modo que el menor pueda sentirse cómodo y establecer un buen rapport con su entrevistador al principio de la entrevista. Después, cuándo estos protocolos se están utilizando con el objetivo de valorar la credibilidad del testimonio del menor, se van a utilizar siempre técnicas de recuerdo para valorar las capacidades del menor y la capacidad de discernir entre la verdad y la mentira. Una vez realizado el entrenamiento en memoria y habiendo valorado las capacidades del menor, su lenguaje y su ubicación espaciotemporal, se debe introducir el tema del abuso con preguntas abiertas y evitando siempre la sugestión, para después pasar al recuerdo libre de los hechos por parte del menor. Con menores se recomienda también el uso de preguntas aclaratorias para recabar detalles concretos que puedan haber sido obviados en su discurso sobre los hechos. Al terminar, siempre hay que darle la oportunidad al menor de preguntar cualquier cosa que pueda haber quedado en duda o de aportar cualquier dato que le haya quedado pendiente, pero es necesario que el entrevistador termine tratando un tema trivial para retirar el foco de atención del menor sobre el asunto traumático y llevarle a un estado emocional neutro (Vizcarra et al., 2018).

Además de estas características comunes, los diferentes protocolos presentan algunas variaciones de unos a otros, por ejemplo: la Entrevista Paso a Paso o Step Wise Interview destaca por aplicar las ideas de la psicología evolutiva a la entrevista gradual con el objetivo de obtener la máxima información concreta sobre el suceso, adaptando la entrevista al desarrollo y las capacidades del menor; el Protocolo NICHD propone una estructura de entrevista más estructurada pero siempre con preguntas abiertas; el Protocolo Michigan incorpora una fase de preparación previa a la narrativa libre, para garantizar la validez de la información obtenida; y, por último, el Protocolo RATAC-SATAC destaca por su flexibilidad, presta especial atención a las características cognitivas, sociales y emocionales del niño, priorizando la individualización y el uso de recursos que permitan adaptar el curso de la entrevista a las necesidades de cada niño, como por ejemplo muñecos o dibujos. 
El aspecto más importante a la hora de realizar una entrevista a una víctima es evitar la revictimización. La correcta atención multidisciplinar a la víctima desde las diferentes instituciones implicadas será aquella que contribuya decisivamente a minimizar cualquier posible daño secundario en la interacción (Nielsen et al., 2016).

Desde aquí, la entrevista con víctimas exige unas actitudes del evaluador y unas condiciones de relación determinadas que la diferencian de cualquier otro tipo de entrevista de evaluación, por ejemplo, la necesidad de que la víctima se sienta reconocida y validada en todo momento (Greeson, Campbell y Fehler-Cabral, 2016).

Los protocolos o guías existentes para asegurar una buena praxis a la hora de realizar entrevistas a víctimas y testigos que hemos visto responden a la preocupación global actual por minimizar los perjuicios asociados a las entrevistas investigativas para las víctimas sin perder información relevante para el procedimiento (Maffioletti, 2011). Prácticamente todos los autores coinciden en que, para empezar, siempre que sea posible es fundamental grabar la entrevista. Esto permite disponer del contenido literal, revisar posibles errores en el desempeño y evita que la víctima sea expuesta reiteradas veces a la obligación de tener que relatar los hechos sucedidos (Vasquez, 2005).

La mayoría de los autores coinciden en la importancia del acompañamiento a la víctima, la explicación clara sobre todo el proceso que se va a llevar a cabo y las funciones de los profesionales que participan en él, así como la adecuación en cada caso a la realidad de las víctimas y las particularidades de su propia experiencia así como el asesoramiento y orientación en la toma de decisiones, entre otras medidas fundamentales que se deben garantizar para evitar la victimización secundaria en el trato con víctimas de graves traumas como es el abuso sexual infantil (Dos Reis et al., 2016; Maffioletti, 2011). Por todo ello es necesario que la entrevista tenga flexibilidad para adaptarse a las necesidades y demandas, tanto explícitas como implícitas, de cada víctima particular (Burgess y Holmstrom, 1974).

Solo cuando el profesional ha conseguido que la víctima se sienta entendida, escucha y tenida en cuenta es cuando va a poder ayudarla.

\section{DISCUSIÓN Y CONCLUSIONES}

El abuso sexual infantil (ASI) es un problema de especial gravedad en el panorama mundial. A pesar de que muchos casos permanecen ocultos, numerosos informes y estudios revelan cifras tan significativas convierten el 
abuso sexual infantil en un fenómeno especialmente relevante en la realidad actual.

El ASI supone una experiencia traumática que siempre va a tener consecuencias negativas para quién lo sufre, tanto en el nivel físico, psicológico y social.

No es tan frecuente que los efectos del ASI se prolonguen en el largo plazo, ya que esto sólo sucede cuando la víctima no es capaz de superar el trauma sexual a través de un proceso de reparación y reorganización de la experiencia. A lo largo de la bibliografía se identifican distintas variables que van a determinar la intensidad y evolución de la sintomatología en las víctimas.

Para la evaluación con víctimas existen distintos protocolos de entrevista que permiten obtener la máxima información causando el menor daño posible, ya que la máxima de toda intervención con víctimas debe ser siempre evitar la revictimización. Sin embargo, a pesar de que estos modelos ofrecen orientaciones que pueden servir de ayuda para organizar y estructurar la entrevista y acerca de qué preguntar, qué no y cómo hacerlo, trabajando con una población especialmente vulnerable como son las víctimas de ASI, será necesaria también una formación especializada de los profesionales involucrados, una serie de aptitudes y actitudes básicas, así como la práctica o experiencia en el trato con víctimas para garantizar una intervención profesional lo menos perjudicial posible.

Aquí es importante destacar algunos elementos que van a marcar la diferencia entre una entrevista de evaluación psicológica general frente las entrevistas de evaluación con víctimas.

Una entrevista de evaluación es un proceso estructurado y replicable, sin embargo, cuando se trata de víctimas el rasgo diferencial de la entrevista es que cada experiencia es única y particular y por tanto no existe un mismo procedimiento aplicable a todos los casos. Mientras que la entrevista de evaluación simplemente se ajusta al marco de referencia más adecuado para la persona que se está entrevistando en cada caso, la entrevista con víctimas se caracteriza por la flexibilidad y la adaptación a las características y circunstancias de la persona y su experiencia personal, lo que convierte el proceso en único e irrepetible.

En todo caso hay que ofrecerle a la persona entrevistada toda la información acerca del proceso en el que va a participar, los profesionales involucrados y los pasos que se van a seguir, así como generar un entorno de intimidad y protección que favorezca la espontaneidad y que genere la confianza suficiente para que la persona hable de forma natural sobre su experiencia. Sin embargo, es especialmente relevante que, tratando con víctimas, 
debemos priorizar siempre la atención personal por encima de la obtención de información.

La clave de la actitud del profesional en toda entrevista con víctimas de abuso sexual infantil es la escucha activa. Todas las víctimas de ASI van a sufrir una reacción emocional negativa, pero la experiencia de cada una será siempre única y particular. Por ello, la clave fundamental a la hora de intervenir con estas víctimas es la escucha activa, que permite identificar las variables que han influido en su experiencia tras el trauma sexual, evaluar el impacto emocional y psicosocial y realizar así una asistencia adecuada, individualizada y adaptada a las necesidades y expectativas de cada una.

Podemos decir que, a pesar de que no hay una buena praxis común a la hora de trabajar con víctimas de ASI, sí que hemos identificado algunas necesidades comunes presentes en todas las víctimas: ser creídas, no juzgadas, sentirse protegidas, sentir que tienen el control sobre la situación y ser capaces de verbalizar su experiencia, de ponerla en palabras. Esta verbalización de lo ocurrido a través de la entrevista lleva asociadas distintos beneficios para las víctimas. Compartiendo su experiencia la víctima tiene la oportunidad de recibir puntos de vista distintos o nuevas perspectivas que ayudan a la resignificación de la experiencia, además es una vía de desahogo y para sentirse escuchada, reconocida y apoyada, además de abrir la posibilidad a que se produzca lo que en psicología se conoce como insight, que es el proceso de escucharse a uno mismo y reconocer la experiencia que tenemos de nuestras propias vivencias.

No debemos olvidar que en casos de ASI es muy común también que los miembros de la familia y del entorno social más cercano a las víctimas puedan necesitar también una atención especializada.

La entrevista con víctimas de ASI debería estructurarse siempre de la siguiente manera:

Primero, es necesaria una fase previa de preparación u orientación, en la que el profesional lleva a cabo un trabajo de documentación y conocimiento de las características y circunstancias individuales de la persona la que se va a entrevistar. Es necesario conseguir toda la información posible respecto de la experiencia concreta y particular de la víctima para poder adaptar la entrevista a sus necesidades y expectativas. También, este conocimiento nos permite predecir el patrón de comportamiento de la persona y, en consecuencia, anticiparnos a sus reacciones o posibles respuestas y saber cómo acercarnos a ella para lograr la colaboración en la entrevista, elemento fundamental y determinante del éxito o fracaso de esta.

La entrevista comienza con la presentación por parte del profesional, siempre acompañada de una explicación de lo que se va a realizar, si se va 
a grabar la entrevista o no, cuál es la finalidad de la entrevista, y si van a intervenir otros profesionales, entre otros aspectos esenciales del proceso.

$\mathrm{Al}$ inicio de la entrevista es cuando se busca establecer el vínculo, alianza o buena relación entre entrevistado y entrevistador. Con víctimas de ASI es necesario que el entrevistador transmita una actitud de absoluto respeto, que se debe reflejar a través de conductas básicas como atender a lo que la víctima está contando, observar lo que transmite a nivel de conducta no verbal y reconocer el valor de la persona y su dignidad, para que se sienta siempre reconocida y protegida. En esta fase de la entrevista es recomendable realizar preguntas que denoten interés por la persona en sí y no por la experiencia traumática de la que queremos hablar.

En este punto habría que realizar lo que se conoce como un entrenamiento en un episodio de memoria, de modo que se le pide a la víctima que relate alguna experiencia o episodio de su pasado.

Una vez establecido el vínculo y generado un ambiente de confianza, el entrevistador debe utilizar preguntas exploratorias para introducir el tema del abuso sexual en concreto. Es muy importante que la víctima se sienta cómoda para poder hablar de forma espontánea acerca del suceso y, por lo tanto, no convertir la entrevista en un interrogatorio. Aquí es donde toma especial relevancia la necesidad de un entrenamiento por parte de los entrevistadores en la habilidad para formular preguntar exploratorias, de modo que no resulten invasivas ni demasiado directas.

Habrá que combinar las preguntas directas y de clarificación con preguntas abiertas, que deben predominar en la entrevista ya que el objetivo es conseguir que la víctima realice una narración libre. Las preguntas directas se deben utilizar para aclarar cuando haya alguna información confusa. Sin embargo, aunque buscamos la narrativa libre de la víctima, es el profesional que realiza la entrevista quien debe siempre guiar y mantener el control.

El entrevistador debe prestar especial atención a los signos o indicadores de fatiga, ansiedad o estrés en la víctima, para ofrecer un descanso si lo necesita.

Finalmente, la fase de cierre es muy importante en estas entrevistas. Aquí se debe siempre agradecer a la víctima su confianza y esfuerzo por compartir su experiencia y, más importante aún, se le da la oportunidad de decir cualquier cosa que le haya quedado pendiente o que quiera añadir, así como de preguntarnos cualquier posible duda.

Toda entrevista exige una fase final de interpretación de la información por parte del profesional, desde el marco individual de la persona, en la que se procede a relacionar la información obtenida con las características de su experiencia. 
Hay mucho escrito acerca del abuso sexual infantil y la experiencia de las víctimas al participar en procesos de evaluación, de modo que es inevitable que hayan quedado fuera de esta revisión multitud de materiales relevantes que avalen lo que aquí se discute o incluso abran la puerta a alguna otra alternativa o propuesta diferente. La principal limitación de este estudio es que el filtro por idioma (español e inglés) va a suponer un sesgo cultural que reduce la visión global del tema abordado. Además, por tratarse de un diseño retrospectivo, se centra en la revisión de artículos y estudios anteriores por lo que podría ser una limitación para mejorar en futuros estudios el proponer una mirada más orientada a las oportunidades de intervención que ofrecen los avances tecnológicos, por ejemplo.

A pesar de estas limitaciones, el estudio cumple con los objetivos perseguidos de identificar los principales efectos asociados al abuso sexual infantil, así como las claves a la hora de evaluar a una población tan vulnerable como son las víctimas de abuso sexual infantil, identificando también particularidades que diferencian este tipo de evaluación de cualquier entrevista de evaluación. La revisión llevada a cabo recoge los aspectos más importantes a tener en cuenta para evitar la revictimización en el trato con víctimas, lo que abre la posibilidad a futuros investigadores a poner en práctica las claves identificadas y evaluar empíricamente su utilidad, para poder perfeccionar las técnicas de intervención e investigación en este campo.

\section{REFERENCIAS}

Arce, R., y Fariña, F. (2012). La entrevista psicológica forense a niños, adultos y discapacitados. En S. Delgado, F. Bandrés y A. Tejerina (Coords.). Tratado de medicina legal y ciencias forenses: Vol V. Pediatría legal y forense (pp. 795-817). Barcelona: Editorial Bosch.

Burgess, A. W., y Holmstrom, L. L. (1974). Crisis and counseling request of rape victims. Nursing Research 23(3), 196-202.

Cantón-Cortés, D., y Justicia, F. (2008). Afrontamiento del abuso sexual infantil y ajuste psicológico a largo plazo. Psicothema, 20(4), 509-515.

Cantón-Cortés, D., y Cortés, M. R. (2015). Consecuencias del abuso sexual infantil: una revisión de las variables intervinientes. Anales de Psicología, 31(2), 552-561.

Carrasco, J. J., y Maza, J.M. (2005). Manual de psiquiatría legal y forense. Madrid: La Ley.

Centros de Servicios Sociales y Servicio especializado en Menores (2014). Protocolo de actuación en la Comunidad Autónoma de Aragón. La detección y notificación en casos de abuso sexual infantil en Aragón. Aragón: Servicio Especializado en Menores. 
De la Cruz, M. A. (2014). Factores predictores del impacto psicopatológico en víctimas de agresión sexual. Madrid, España: Ministerio de Salud, Servicios sociales e Igualdad.

Dos Reis, M. J., Baena de Morales Lopes, M. H., y Duarte Osis, M. J. (2016). «It's much worse than dying»: the experiences of female victims of sexual violence. Journal of Clinical Nursing, 26(15-16), 2353-2361.

Duma, S.E, Mekwa, J.N., y Denny, L.D. (2007). Women's journey of recovery from sexual assault trauma: a grounded theory - part 1. Curations 30, 4-11.

Echeburúa, E., Corral, P., Sarasua, B., Zubizarreta, I., y Sauca. D. (1994). Entrevista semiestructurada para víctimas de maltrato doméstico. En E. Echeburúa, y P. Corral (Eds.). (2006). Manual de violencia familiar. Madrid: Siglo XXI.

Echeburúa, E., y del Corral, P. (2006). Secuelas emocionales en víctimas de abuso sexual en la infancia. Cuadernos de Medicina Forense, 12(43-44), 75-82.

Fear, R. A. (1979). La entrevista de evaluación. Buenos Aires, Argentina: Paidos.

Fernández-Ballesteros, R. (2004). Evaluación psicológica. Conceptos, métodos y estudios de casos. Madrid, España: Pirámide.

Filipas, H. H., y Ullman, S. E. (2006). Child sexual abuse, coping responses, selfblame, posttraumatic stress disorder and adult sexual revictimization. Journal of Interpersonal Violence, 21, 652-672.

Fisher, R. P., y Geiselman, R. E. (1992). Memory-enhancing Techniques for Investigative Interviewing: The Cognitive Interview. Springfield, Illinois: Charles C. Thomas.

González Fernández, J., y Pardo Fernández, E. (2007). El daño psíquico en las víctimas de agresión sexual. Trabajo publicado en el VII Congreso Virtual de Psiquiatría.

Greeson, M. R., Campbell, R., y Fehler-Cabral, G. (2016). «Nobody deserves this»: Adolescent sexual assault victim's perceptions of disbelief and victim blame from police. Journal of Community Psychology, 44(1), 90-110.

Lama, X., Gutiérrez, C., Capella, C., Dussert, D., Rodríguez, L., Beiza, G., y Águila, D. (2015). Superación de agresiones sexuales infanto-juveniles y el sistema judicial: una visión desde los participantes. Revista de Psicología, 24(1), 1-19.

López, F. (1995). Prevalencia y consecuencias del abuso sexual al menor en España. Child Abuse and Neglect, 19(9), 1039-1050.

López, F. (1996). Abusos sexuales a menores: lo que recuerdan de mayores. Madrid, España: Ministerio de Asuntos Sociales.

Maffioletti, F. (2011). La entrevista forense a la víctima de delitos sexuales. Revista Jurídica del Ministerio Público, 38, 199-228.

Mas, B. (1995). Trastorno por estrés postraumático: el abuso sexual infantil y su tratamiento. En J. M. Buceta y A. M. Bueno (dirs.). Psicología y salud: control del estrés y trastornos asociados (pp. 385-420). Madrid: Dykinson.

Mercado Justiniano, G. Z. (2017). Criminología Victimal. La revictimización procesal de la agresión sexual y sus consecuencias neuropsicoemocionales: investigación preliminar y reacción a los medios sociales. Archivos de Criminología, Seguridad Privada y Criminalística, 9, 94-104.

Muñoz, J. M. (2013). La evaluación psicológica forense del daño psíquico: propuesta de un protocolo de actuación pericial. Anuario de Psicología Jurídica, 23, 61-69. 
Muñoz, J. M., González-Guerrero, L., Sotoca, A., Terol, O., González, J. L., y Manzanero, A. L. (2016). La entrevista forense: obtención del indicio cognitivo en menores presuntas víctimas de abuso sexual infantil. Papeles del Psicólogo, 37(3), 205-216.

Nielsen, L. H., Hansen, M., Elklit, A., y Bramsen, R. H. (2016). Sexual assault victims participating in research: causing harm when trying to help? Archives of Psychiatric Nursing, 30(3), 412-417.

Pereda, N. (2016). ¿Uno de cada cinco? Victimización sexual infantil en España. Papeles del Psicólogo, 37(2), 126-133.

Pereda, N., y Gallardo-Pujol, D. (2011). Revisión sistemática de las consecuencias neurobiológicas del abuso sexual infantil. Gaceta Sanitaria, 35(3), 233-239.

Turcios Morales, L. A. (2012). Estudio descriptivo del abuso sexual infantil en El Salvador durante el periodo 2001-2009. Recuperado de Irradia terapia México: http://psicologos.mx/estudio-sobre-abuso-sexual-infantil.php

Shin, K. M., Chang, H. Y., Cho, S. M., Kim, N. H., Kim, K. A., y Chung, Y.K. (2015). Avoidance symptoms and delayed verbal memory are associated with post-traumatic stress symptoms in female victims of sexual violence. Journal of Affective Disorders, 184, 145-148.

Stiller, A., y Hellmann, D. F. (2017). In the aftermath of disclosing child sexual abuse: consequences, needs and wishes. Journal of Sexual Aggression, 23(3), 251-265.

Ullman, S. E., Najdowski, C. J., y Filipas, H. H. (2009). Child sexual abuse, posttraumatic stress disorder, and substance use: Predictors of revictimization in adult sexual assault survivors. Journal of Child Sexual Abuse, 18, 367-385.

Ullman, S. E., y Vasquez, A. L. (2015). Prevalence and revictimization research for victims of child sexual abuse. Mediators of Sexual Revictimization Risk in Adult Sexual Assault Victims. Journal of Child Sexual Abuse, 24, 300-314.

Vázquez, B. (2005). Manual de Psicología Forense. Madrid: Síntesis.

Vizcarra, W. A., Marín, F. C., y Díaz, S. R. (2018). La entrevista a Víctimas y/o Testigos en las Áreas de Psicología y Trabajo Social de la Unidad de Protección a Víctimas y Testigos (UPAVT). Bolivia: Ministerio Público, Fiscalía General del Estado. 